\title{
Integração do Índice de Irregularidade Internacional (IRI) em um SIG para análise espacial da qualidade do pavimento
}

\author{
Alessandra dos Santos Mendes e Arley Ferreira de Souza \\ Faculdade de Tecnologia de Jacareí - FATEC Jacareí. Av. Faria Lima, Jd. Santa \\ Maria - Jacareí - SP, 12.328-150. (alessandra.s.mendes@hotmail.com, \\ arley.souza@fatec.sp.gov.br)
}

\begin{abstract}
Resumo: Este trabalho apresenta o ambiente SIG como ferramenta para análise espacial do Índice de Irregularidade Internacional (IRI) a fim de avaliar a qualidade do pavimento. A área de estudo refere-se à Rodovia Presidente Dutra, km 0 ao km 37, faixas 1 e 2, pista sul, regional São Paulo.O sistema de medida utilizado para a aquisição dos dados de IRI foi um perfilômetro inercial laser, modelo CiberLaser, desenvolvido pela empresa brasileira CiberMétrica Ltda.Os resultados apresentados nesta pesquisa foram alguns exemplos de aplicações de um ambiente de (SIG) nos dados de qualidade de pavimento, entre eles: visualização espacial, comparação e associação entre dados e potencialidades das operações espaciais.Durante a pesquisa o SIG apresentou ser uma ferramenta promissora capaz de melhorar a percepção visual sobre os dados e por consequência facilitar no processo de tomada de decisões.
\end{abstract}

Palavras chave: Sistema de Informação Geográfica, Índice de Irregularidade Internacional,perfilômetro, gestão de pavimento

\begin{abstract}
This work presents the Geographic Information Systems environment (GIS) as a tool to conduct spatial analysis of the International Roughness Index (IRI) in order to evaluate pavement quality. The study area refers to Dutra Highway ( $\mathrm{Km} 0$ to $\mathrm{Km} \mathrm{37,}$ lanes 1 and 2, southbound in São Paulo). The utilized measurement system to obtain the III data was a laser inertial profilometer model Ciberlaser, developed by the Brazilian company CiberMétrica Ltd. The presented results in this research came from a few application examples of a GIS environment concerning the pavement quality data including spatial visualization, comparison and data association and spatial operation potentialities. During the research, the GIS revealed to be a promising tool able to improve the visual perception on the data so as to make the decision making process easier.
\end{abstract}

Key Words: Geographic Information System, International Roughness Index, profilometer, pavement management

\section{Introdução}

A avaliação dos pavimentos é uma das etapas mais importantes por ser o ponto de partida para futuras decisões em um Sistema de Gerência de Pavimentos (SGP). Esta atividade possibilita que sejam estudadas as condições estruturais e funcionais dos pavimentos, adotando-se os procedimentos e as metodologias que sejam melhores apropriados às características dos trechos de uma malha viária (BENEVIDES, 2008). 
Segundo Rodrigues (2013), com o objetivo de obter estimativas para parâmetros ligados à degradação de pavimentos, é importante que um SGP possibilite a qualificação da malha através de um indicador, tal como a irregularidade longitudinal do pavimento (VOGEL, 2015). Para entendimento, a irregularidade longitudinal é o conjunto dos desvios da superfície de um pavimento em relação a um plano de referência. Esses desvios afetam diretamente a dinâmica do veículo e das cargas sobre a via, a qualidade do rolamento e ainda a capacidade de drenagem superficial (CNT, 2017).

Em 1982, o Banco Mundial conduziu uma pesquisa no Brasil conhecida como International Road Roughness Experiment (IRRE), realizado por equipes de pesquisa do Brasil, Inglaterra, França, Estados Unidos e Bélgica. Quarenta e nove (49) locais de teste foram medidos usando uma variedade de equipamentos e condições de medição. Assim, propôs-se um único índice, denominado International Roughness Index (IRI). O IRI é mensurável por todos os equipamentos de medição de rugosidade incluídos no IRRE, e também é compatível com quase todos os equipamentos utilizados em todo o mundo (SAYERS et al., 1986).

No Brasil, a escala padrão de medição adotada é o Quociente de irregularidade (QI ou Índice de Quarto de Carro), reconhecido internacionalmente. Os conceitos de QI e IRI são bastante similares e, na prática, eles são altamente correlacionados. Uma relação aproximada entre QI e IRI é dada por: $\mathrm{QI}=13 \mathrm{IRI}$ (DNIT, 2006).

As análises de $\mid \mathrm{RI}$ são basicamente relações estatísticas, ou seja, tendem a desprezar fatores de correlação espacial que são de grande relevância para avaliação do pavimento, tais como: obras de arte, drenagens, dispositivos de segurança, volume de tráfego, radares, entre outros.

Devido ao fato de que as agências de transporte têm acumulado imensas quantidades de dados sobre a condição do pavimento, SIG (Sistema de Informação Geográfica) tornou-se uma ferramenta útil para esses sistemas de gestão. Isso tornou imperativo que as agências encontrem uma maneira de armazenar e gerenciar primeiro uma quantidade tão grande de dados e, em segundo lugar, ter a capacidade de usar efetivamente esses dados para tomar decisões apropriadas e de custo eficaz em relação à manutenção e reabilitação de pavimentos (GRASS, 2007).

Elhadi (2009) cita estudo cujo objetivo era avaliar a condição do pavimento das estradas existentes através da realização de dois tipos de ensaios, o teste de rugosidade para a determinação do IRI e o teste de deflexão não destrutivo, particularmente o método de deflexão de queda de peso. Os dados coletados foram inseridos como atributos no ArcGIS, e uma extensão do software conhecida como VideoDRS, também foi usada para habilitar a indexação e reprodução do vídeo em relação aos pontos de GPS. Neste estudo, concluiu-se que o SGP baseado em SIG fornece um meio eficiente para identificar padrões empíricos e relacionamentos que podem não ser detectados usando métodos estatísticos e padrões descritivos. A exibição 
gráfica das tendências de dados é um método eficaz de comunicação entre o sistema e o usuário. O SIG não se destina a substituir a perícia de engenharia e julgamento, mas sim para ajudar na tomada de decisões rentáveis (ELHADI, 2009).

Ibraheem (2012) descreve um trabalho no qual foram selecionadas 23 secções das estradas da Universidade de Nahrain, Bagdá/Iraque, com problemas de diferentes causas como intempérie, envelhecimento, carga de tráfego e também a má manutenção. Ibraheem, conclui que o SIG é uma escolha lógica para SGP, pois provou ser uma ferramenta eficaz para integrar, gerenciar, armazenar, exibir, mapear, consultar e analisar espacialmente dados de transporte (IBRAHEEM, 2012).

Neste contexto, ao usar essas ferramentas de análise espaciais esperase entender melhor o comportamento do IRI e assim identificar padrões não antes detectados. Além disso, ressalvar aos especialistas em SGP e setores de transporte de como o SIG pode ser uma ferramenta promissora capaz de melhorar essa percepção visual sobre os dados e por consequência facilitar no processo de tomada de decisão.

\section{Materiais e Métodos}

Área de estudo: A área de estudo refere-se à Rodovia Presidente Dutra, faixas 1 e 2 da pista sul, regional São Paulo, entre os quilômetros 0 ao 37 (Figura 1).

Figura 1 - Área de estudo. Representação da Faixa 1 da pista sentido Sul da Rodovia Presidente Dutra.

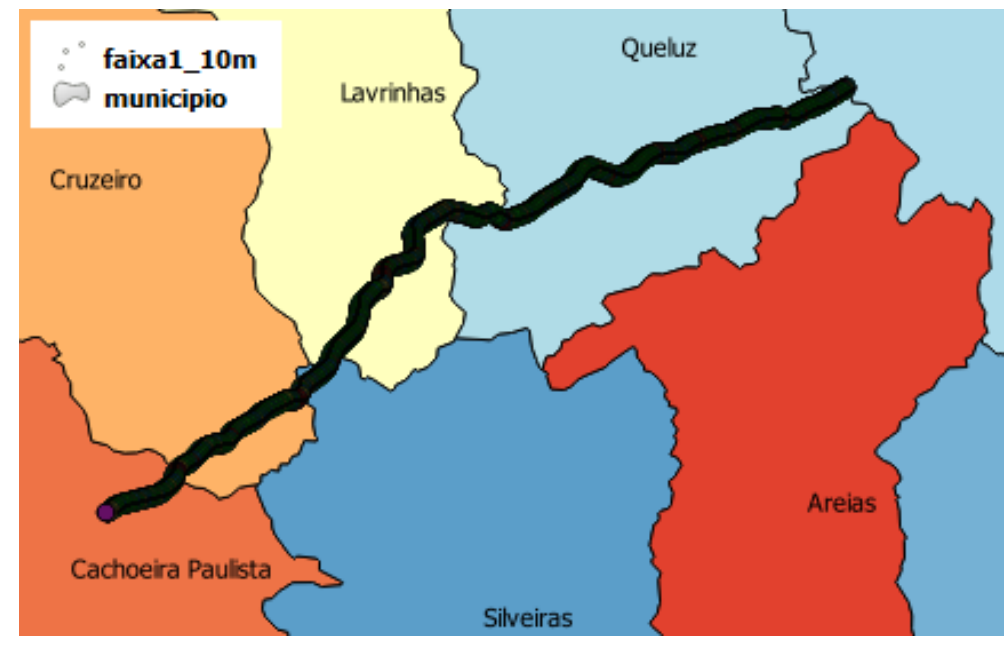

Procedimentos para a coleta de dados: As amostras para o desenvolvimento desse estudo são os dados de IRI do trecho paulista da Rodovia Presidente Dutra, ano 2016, fornecidos pelo Centro de Pesquisas Rodoviários (CPR) CCR Nova Dutra. 
O sistema de medida utilizado para a aquisição destes dados foi o perfilômetro inercial laser, modelo "CiberLaser", desenvolvido pela empresa brasileira CiberMetrica Ltda., assim como mostra a Figura 2.

Este equipamento é destinado à avaliação de Irregularidade Longitudinal de Pavimentos (QI e IRI) que opcionalmente também permite a avaliação dos Afundamentos Plásticos em Trilhas de Roda (ATR). (CIBERMÉTRICA,2017)

Figura 2 - Perfilômetro inercial laser instalado no veículo.

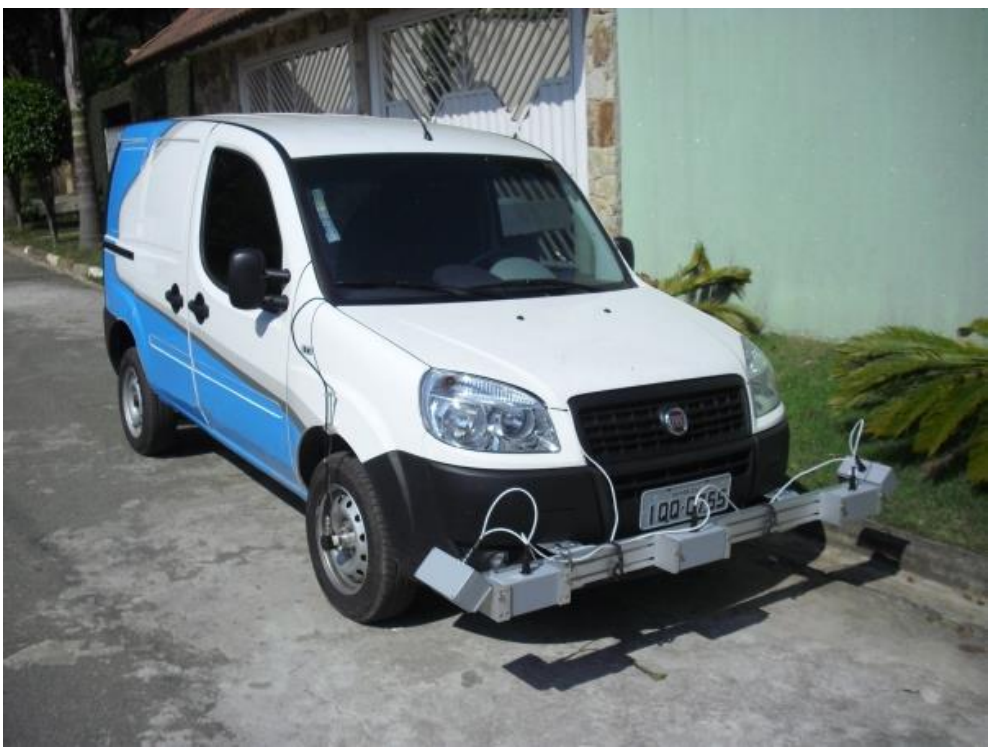

Fonte: CIBERMÉTRICA (2017).

A Figura 3 e Figura 4 ilustram o esquema de funcionamento do perfilômetro inercial no veículo. Ele é constituído de um sistema de módulos laser de medição verticais, módulos laser de medição diagonal (especiais para avaliação de ATR), acelerômetros (medidores de aceleração vertical do veículo), hodômetro de precisão (medidor de distância em veículos), GPS para gravação do posicionamento do veículo, e um Hub que concentra as informações dos componentes e as envia para um computador portátil.

Figura 3 - Ilustração lateral da instalação do perfilômetro inercial no veículo.

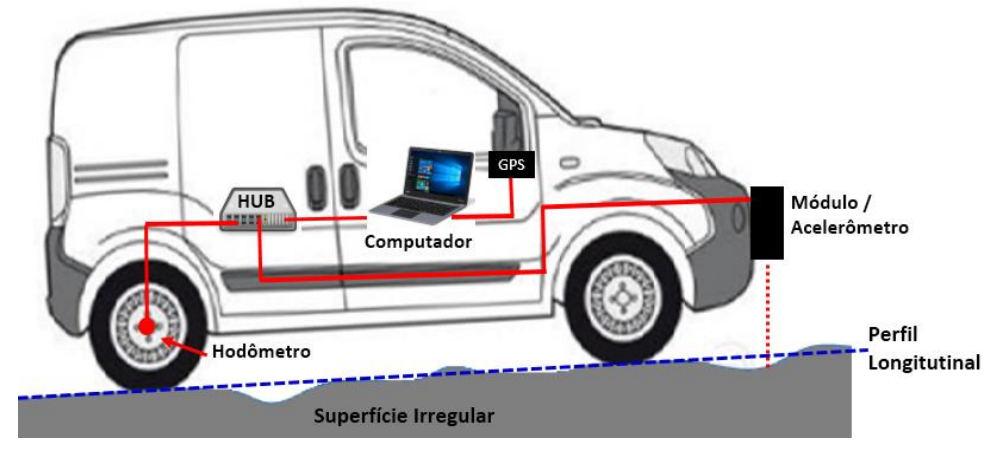


Figura 4 - llustração frontal da instalação do perfilômetro inercial no veículo.

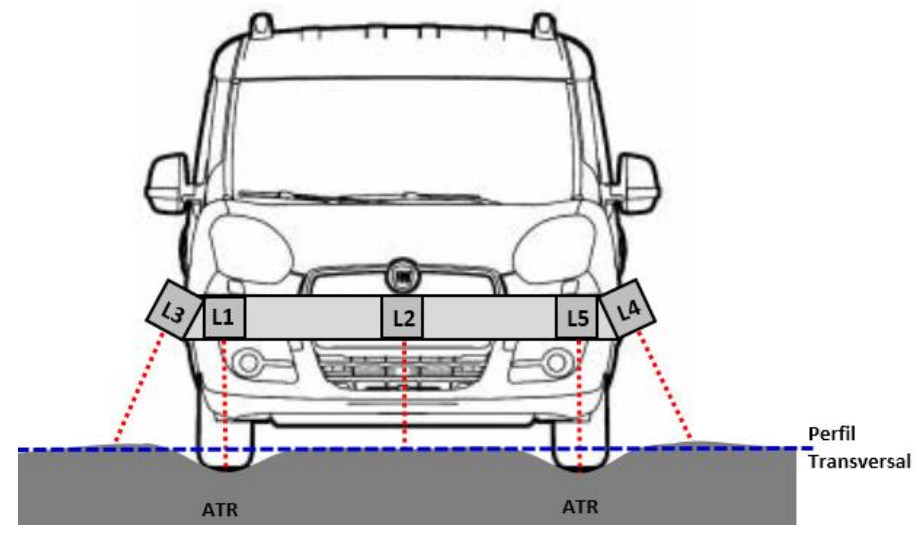

A Figura 5 mostra a interface do software CiberLogger onde o operador acompanha o estado de cada um dos sensores, em tempo real, visando efetuar um controle de qualidade da medição ainda dentro do veículo (Figura 3). A Figura 5 mostra a interface principal durante o levantamento com o CiberLaser.

Figura 5 - Tela principal durante um levantamento com o CiberLaser.

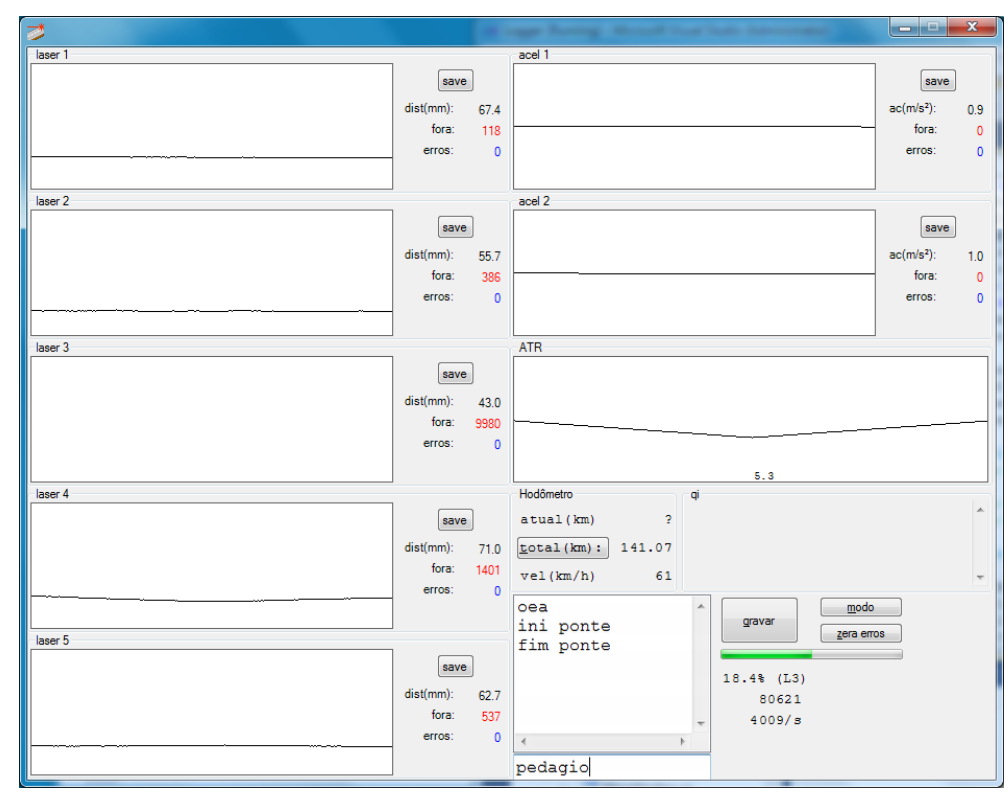

Fonte: CIBERMÉTRICA, 2017.

Para manipular os dados exportados pelo software CiberLogger, os operadores utilizam o software CiberShell (Figura 6). Esse software permite gerar relatórios personalizados que podem conter: os índices IRI, QI e a estimativa do Índice de Perfil (IP), o valor médio dos Afundamentos em Trilhas de Roda (ATR), a velocidade do veículo, as observações de campo, as coordenadas GPS em diversos formatos, incluindo a possibilidade de geração de arquivo KML - compatível com o Google Earth - entre diversas outras funções. (CIBERMÉTRICA, 2017) 
Figura 6 - Tela principal do CiberShell durante o processamento dos dados.

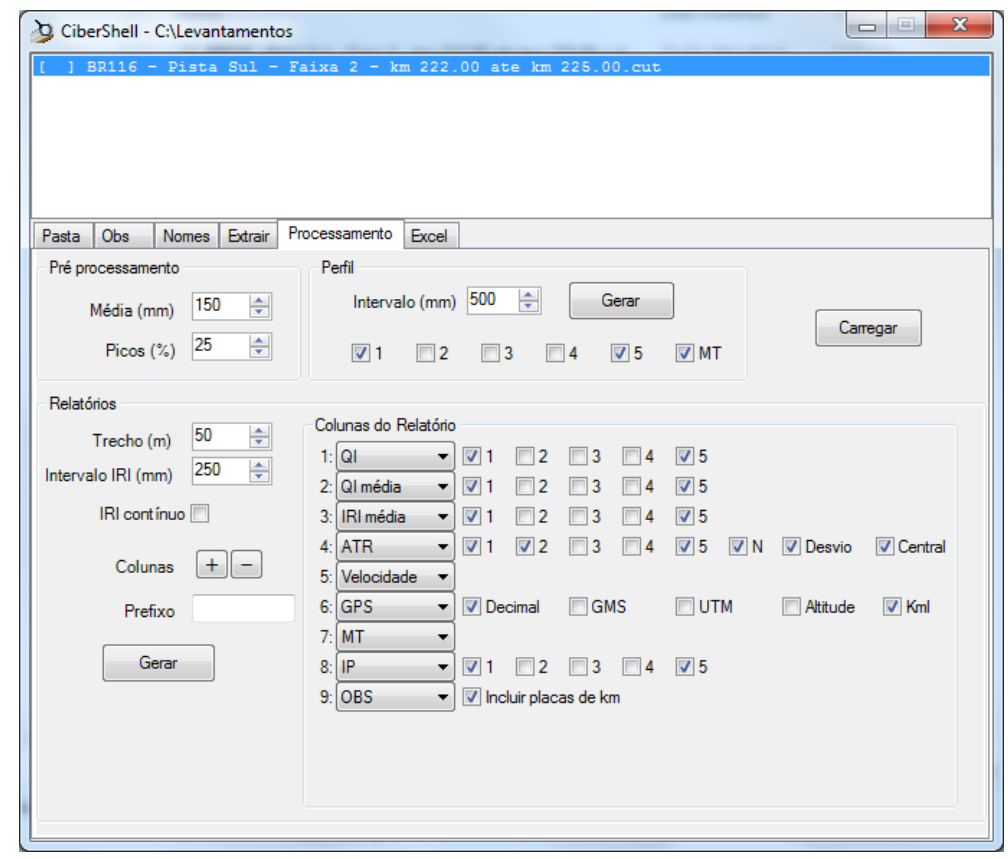

Fonte: CIBERMÉTRICA (2017).

Normas para o processo de levantamento: Conforme especificações técnicas do CPR, este equipamento cumpre todas as especificações e exigências de certificação do DOT - U.S. Departamento Transportation, incluindo AASHTO PP50-02, PP51-02 e Tex 1001- S.Além disso, o levantamento dos dados de irregularidade obedeceu ao procedimento "DNER 159/58 - Projetos de Restauração de Pavimentos Flexíveis e Semi-Rígidos", exigido pela ANTT (Agência Nacional de Transportes Terrestres).

De acordo com o Manual de Restauração de Pavimentos Asfálticos (DNIT 2006), a Irregularidade do Perfil Longitudinal de um Pavimento apresenta as seguintes principais particularidades:

- A partir de um valor inicial, função do processo construtivo, a irregularidade passa a assumir valores crescentes, como decorrência da ação do tráfego, do clima e de outros fatores;

- A irregularidade influi na interação da superfície da via com os veículos, gerando efeitos sobre os próprios veículos, sobre os passageiros e motoristas, e sobre as cargas transportadas. Tais efeitos dependem também, além da irregularidade, de fatores vinculados à suspensão dos veículos e à sensibilidade dos passageiros e das cargas;

- A irregularidade aumenta a ação das cargas dinâmicas dos veículos sobre a superfície do pavimento e, em decorrência, acelera a deterioração de sua estrutura. Ela provoca ainda efeitos adversos sobre a drenagem da superfície do pavimento, na medida em que contribui para a formação de poças d'agua que vão afetar negativamente a segurança e o desempenho da Rodovia; 
- A irregularidade afeta a dinâmica dos veículos em movimento, aumentando o seu desgaste e acarreta, também, prejuízos à condução dos veículos. Em consequência, a irregularidade tem grande influência sobre o custo operacional dos veículos - com o qual apresenta, inclusive, satisfatória correlação estatística.

O perfil longitudinal, por sua vez, inclui o greide, a irregularidade e até a textura do pavimento, dependendo de como e para que ele é medido (Figura 7). Este perfil está relacionado a todos os elementos que caracterizam a boa funcionalidade de um pavimento (SEVERO et al.,2004).

Figura 7 - llustração das condições de irregularidades e texturas no pavimento.

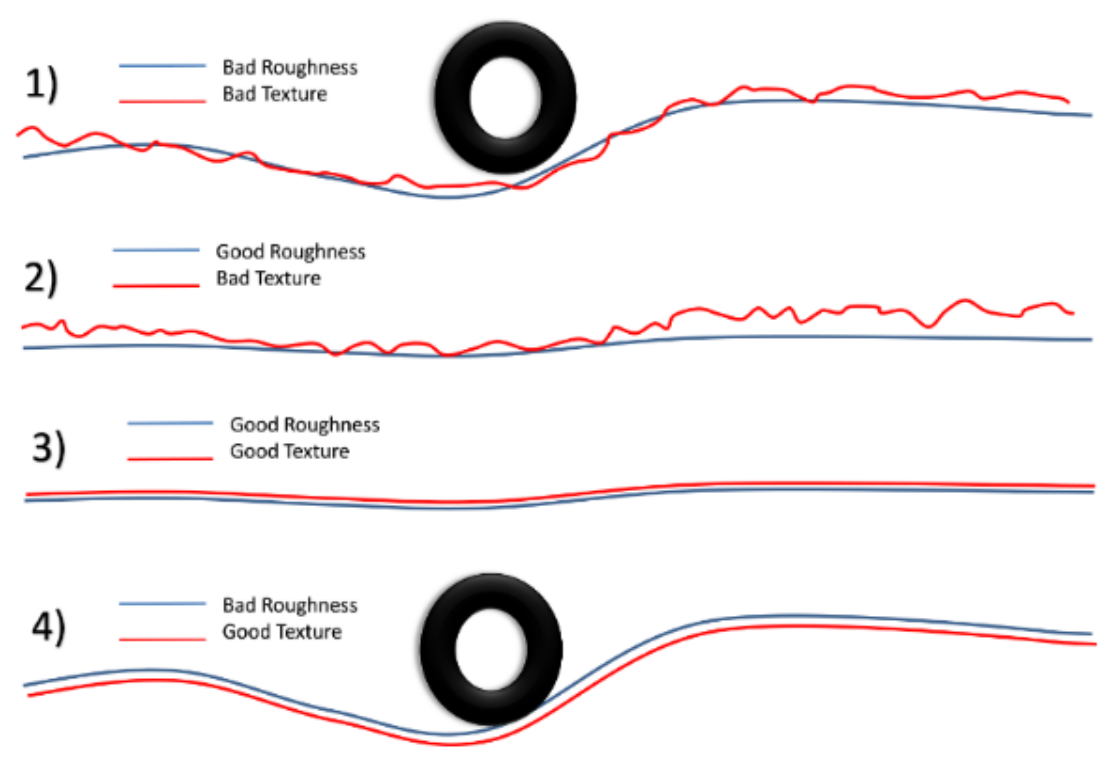

Fonte: FORSLOF (2014).

Quanto à classificação por conceito das condições de superfície do pavimento em função da irregularidade longitudinal medida pelo IRI adotasepara avaliação a norma do DNIT (2006), com valores de IRI agrupados assim como na Tabela 1.

Tabela 1- Classificação das condições de superfície.

\begin{tabular}{|l|l|}
\hline Conceito & IRI $\mathbf{( m / k m )}$ \\
\hline Excelente & $1-1,9$ \\
\hline Bom & $1,9-2,7$ \\
\hline Regular & $2,7-3,5$ \\
\hline Ruim & $3,5-4,6$ \\
\hline Péssimo & $>4,6$ \\
\hline
\end{tabular}

Fonte: DNIT (2006). 
Características dos dados: Os dados de IRI são processados no software CiberShell e exportados no formato de planilhas contendo: valores de IRI agrupados por metros, observações relatadas pelo operador do perfilômetro, identificação do $\mathrm{km}$ e coordenadas geográficas.Como o software permite processar os dados em diferentes trechos (extensão), neste estudo foi utilizado os valores agrupados a cada $10 \mathrm{~m}, 20 \mathrm{~m}$ e $100 \mathrm{~m}$. Esses dados são importados num SIG para serem especializados no formato de um mapa. Neste trabalho foi utilizado o QGIS para manusear os dados.

É importante ressaltar que os dados coletados pelo GPS não conferem com as marcações de $\mathrm{km}$ geradas pelo perfilômetro, já que ele registra 0 deslocamento contínuo do veículo, mesmo quando este se encontra fora da pista, por exemplo, acesso e trevos. Para melhor qualidade do processo de coleta é imprescindível que o operador mantenha o perfilômetro ligado apenas sobre a pista e inicie os percursos numa placa de referência da rodovia.

Quando esses dados são manuseados fora de um SIG, faz-se necessário o ajustamento dos relatos do operador (observações sobre a localização das placas de $\mathrm{km}$, pontes, viadutos, sinalizadores, radares etc.) com os dados da rodovia, pois a posição correta é relevante para a produção e análise do projeto.

Para operar esses dados no SIG foi considerada apenas as coordenadas do GPS.

\section{Resultados}

O ambiente de um SIG possibilita fazer análises tanto visuais quanto espaciais. $\mathrm{Na}$ análise visual o operador pode carregar os dados no SIG e visualizar o resultado como um mapa para fazer as suas considerações. Já usando operações espaciais ele pode fazer cruzamentos dos dados e analisar o resultado da operação.

Avaliação dos valores de IRI de acordo com os intervalos de classificação: $O$ mapa da Figura 8 mostra os valores médios de IRI agrupados em 10 e $20 \mathrm{~m}$. Observa-se que os valores de $20 \mathrm{~m}$ não possuem a mesma sensibilidade comparado aos de $10 \mathrm{~m}$, pois problemas pontuais na superfície do pavimento podem ser mascarados quando os valores de IRI são integrados em extensões maiores.

Os valores de IRI no mapa da Figura 8, bem como ao longo desse trabalho, são classificados de acordo com a Tabela 1. Para melhor visualização dos resultados usou-se as imagens do Google Maps como fundo.

O perfilômetro inercial instalado no veículo, assim como no exemplo da Figura 2, faz leituras da irregularidade da superfície através dos módulos L1, L2, L5 (direito, eixo, esquerdo). Os valores usados na classificação da Figura 8 refere-se apenas a média entre as leituras esquerda e direita de cada ponto de 
Revista Científica e Tecnológica em Meio Ambiente,

GEOHÍDRICA, v.1, n.1: pg. 01-16, 2017.

Recursos Hídricos e Geoprocessamento

Faculdade de Tecnologia de Jacareí -

FATEC Jacarei

Homepage: www.fatecjacarei.com.br

coleta. No exemplo da Figura 8 existem 61 pontos espaçados a cada $10 \mathrm{~m}$ e a Tabela 2 mostra a quantidade de pontos classificados de acordo com as regras da Tabela 1 para as faixas 1 e 2 .

Figura 8 - Valores médios de IRI especializados no mapa no trecho da rodovia entre os $\mathrm{km} \mathrm{26,73}$ ao 27,34 da faixa 1, pista sul.

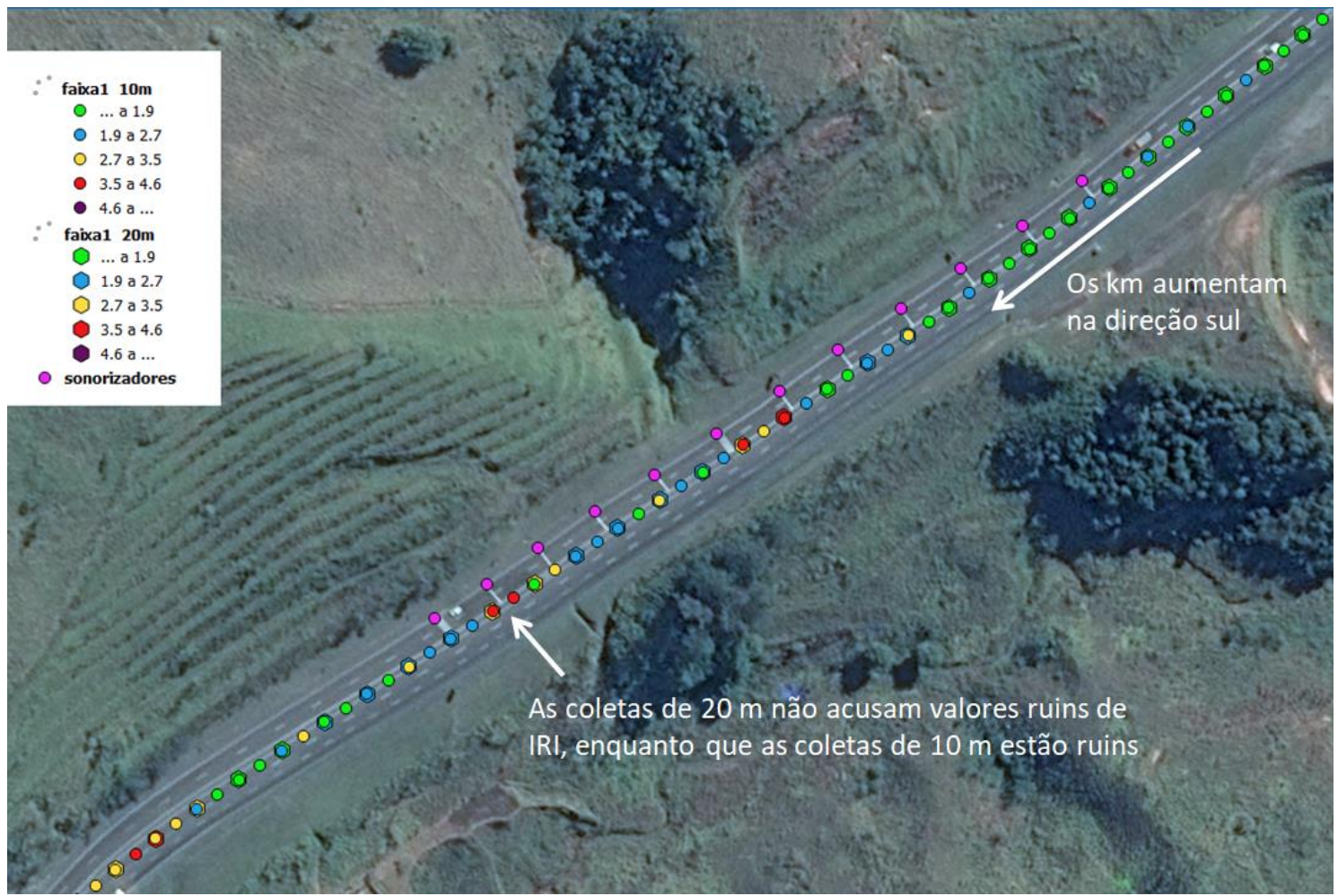

Tabela 2 - Quantidade de pontos classificados de acordo com a norma do DNIT (Tabela 1) no trecho usado como exemplo na Figura 1.

\begin{tabular}{|l|l|l|l|l|l|l|l|}
\cline { 3 - 9 } \multicolumn{2}{c}{} & \multicolumn{2}{l|}{ Faixa 1 } & \multicolumn{2}{l|}{ Faixa 2} \\
\hline Conceito & IRI (m/km) & $\begin{array}{l}\text { IRI } \\
\text { esq. }\end{array}$ & IRI dir. & $\begin{array}{l}\text { IRI } \\
\text { médio }\end{array}$ & $\begin{array}{l}\text { IRI } \\
\text { esq. }\end{array}$ & IRI dir. & $\begin{array}{l}\text { IRI } \\
\text { médio }\end{array}$ \\
\hline Excelente & $\ldots$ a 1,9 & 31 & 25 & 27 & 36 & 41 & 38 \\
\hline Bom & 1,9 a 2,7 & 13 & 23 & 19 & 16 & 15 & 18 \\
\hline Regular & 2,7 a 3,5 & 9 & 10 & 10 & 4 & 3 & 2 \\
\hline Ruim & 3,5 a 4,6 & 8 & 2 & 5 & 4 & 1 & 2 \\
\hline Péssimo & 4,6 a ... & 0 & 1 & 0 & 1 & 1 & 1 \\
\hline
\end{tabular}

Comparação entre os valores esquerdo e direito de IRI: O mapa da Figura 9 mostra os valores de IRI esquerdo e direito da mesma faixa e trecho da 
rodovia do exemplo da Figura 8. A Tabela 2 quantifica os valores de IRI da faixa 1 e 2, das direções (direita e esquerda) e a média de cada ponto. Visualizando o mapa é possível identificar espacialmente onde essas diferenças ocorrem.

Figura 9 - Valores de IRI dos lados esquerdo e direito da faixa 1 da pista sentido sul

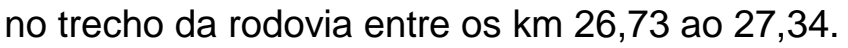

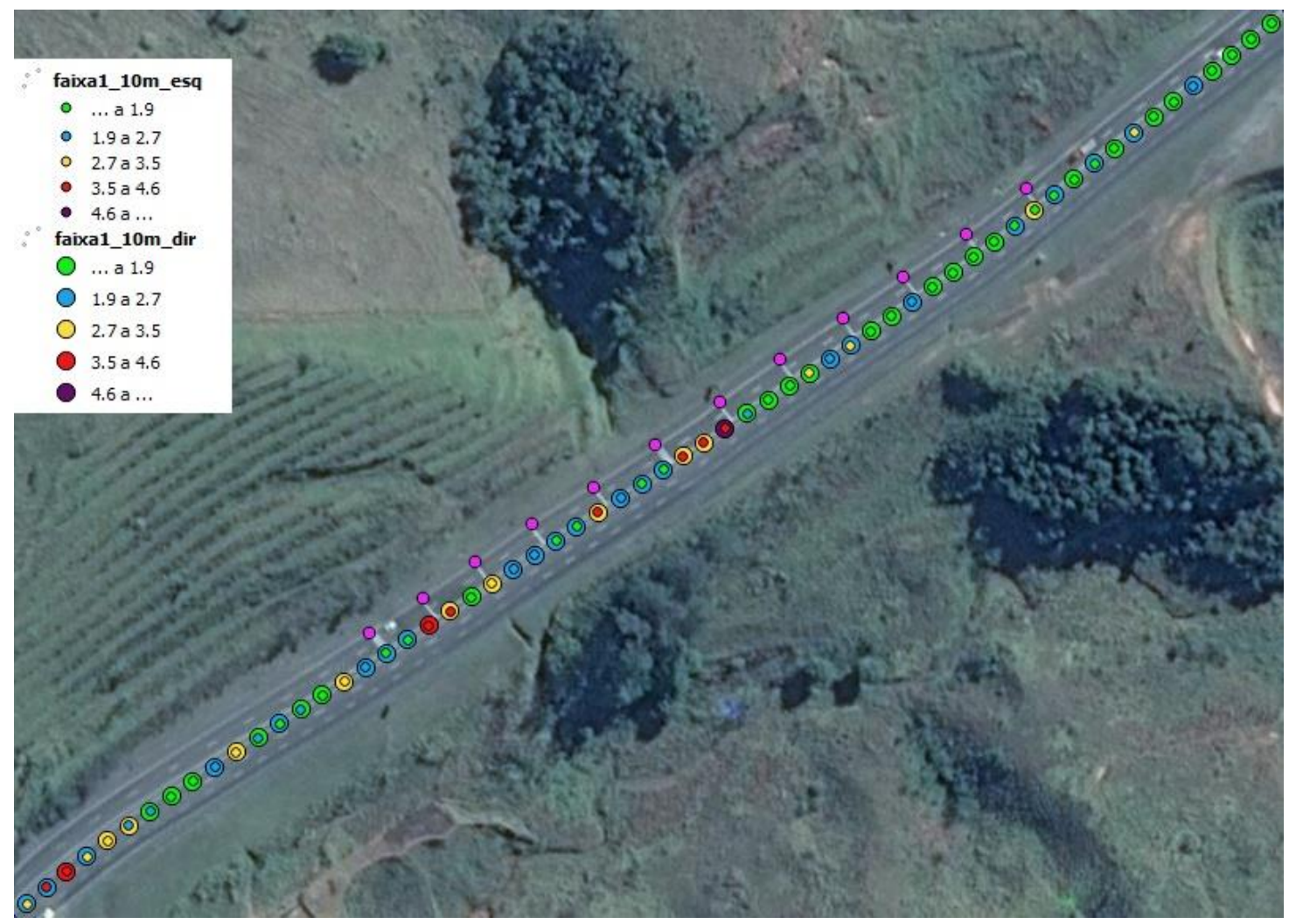

Comparação entre as faixas 1 e 2: Para fazer a comparação serão usados os valores médios de IRI de ambas as faixas espaçados a cada $10 \mathrm{~m}$ no mesmo trecho da rodovia do exemplo da Figura 8. No mapa daFigura 10 estão representados os valores médios de IRI das faixas 1 e 2 . Ao plotar os pontos no mapa é possível visualizar a diferença entre os valores no espaço e assim fazer comparações com outros dados no qual uma simples planilha tabular não é capaz de representar.

Operações espaciais com os dados: Até aqui foram abordados apenas exemplos envolvendo abrir os dados num SIG para plotar os dados no formato de mapa. Porém, os dados podem ser analisados usando operações espaciais.

Nos exemplos a seguir os dados foram carregados no SGBD PostgreSQL 10.1 e as operações espaciais usam a extensão PostGIS na versão 2.4.1. Os dados foram convertidos do sistema de referência espacial WGS84 lat/long para SIRGAS 2000 UTM zona 23S para facilitar as operações 
usando metros. Usando operações espaciais é possível fazer infinitas combinações com os dados. A seguir tem-se três exemplos:

Figura 10 - Valores médios de IRI nas faixas 1 e 2 da pista sentido sul no trecho da rodovia entre os $\mathrm{km} 26,73$ e 27,34 .

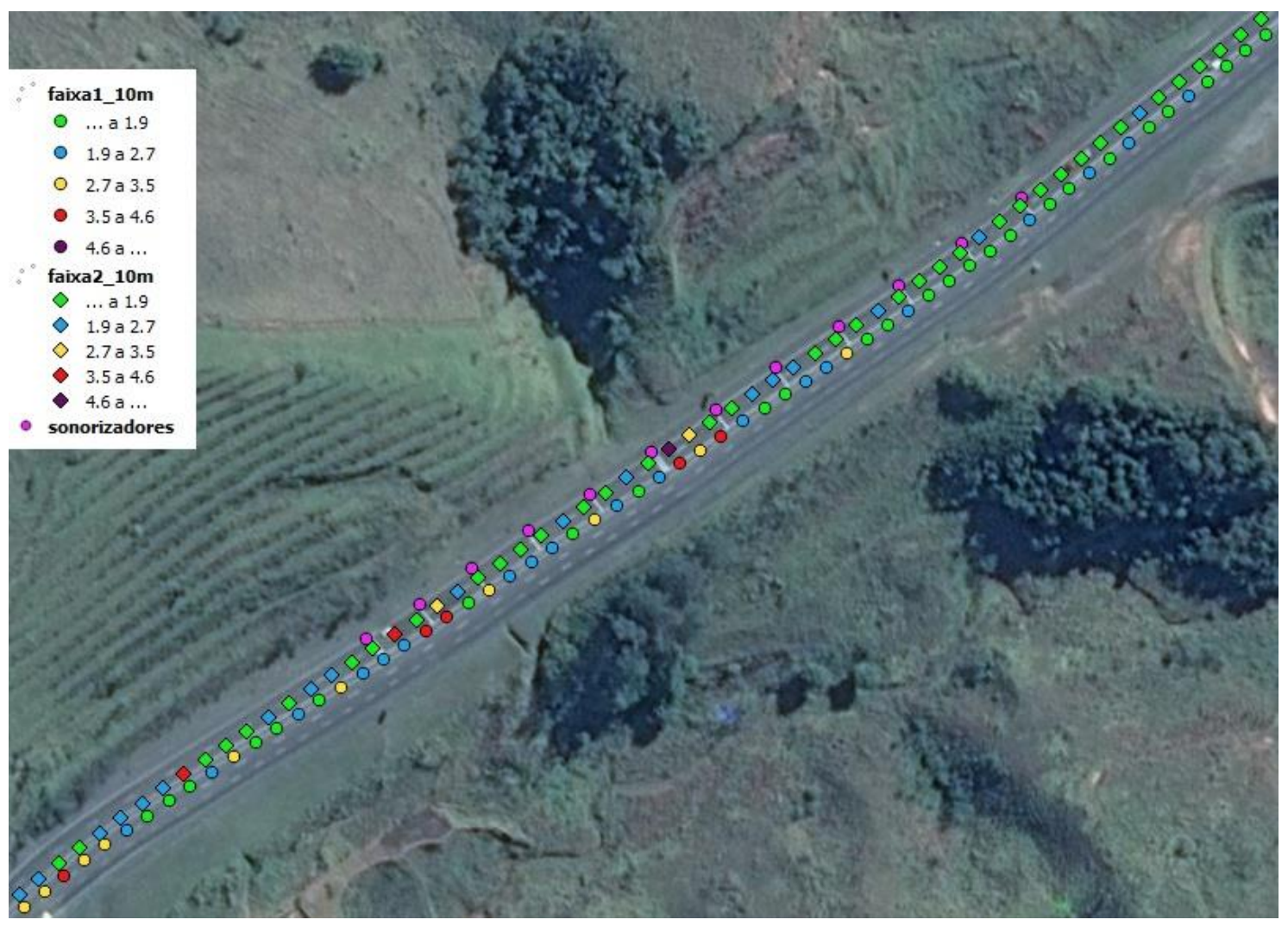

Exemplo 1: Selecionar agrupamento de pontos que estejam na mesma situação, ou seja, que possuem IRI semelhante. Isso possibilita ver que o trecho da rodovia possui algum problema comum. Como estudo de caso, tomou-se a situação de listar os pontos que possuem IRI médio acima de 3,5 e que também possuem algum vizinho a até $21 \mathrm{~m}$ dele, com IRI médio também acima de 3,5. A seguir tem-se a cláusula para fazer essa consulta e na Figura 11 tem-se parte do resultado. Para fazer essa consulta usou-se os dados da faixa 1 espaçados de 10 em 10 metros.

selectdistincton (a.id) a.*

from tbf1_10 as a, tbf1_10 as b

wherea.irimed> 3.5

andb.irimed $>3.5$

and a.id $!=$ b.id

andST_Intersects(a.geom, ST_Buffer(b.geom, 21))

Exemplo 2: Filtrar o resultado do Exemplo 1 (Figura 11) para listar apenas os pontos que estão a até $31 \mathrm{~m}$ de uma OEA. Considerando que o resultado do 
Revista Científica e Tecnológica em Meio

Exemplo 1 foi colocado na tabela tbexemplo1, tem-se a consulta a seguir e o resultado na Figura 12.

select $b . *$

fromtboea_sirgas as a, tbexemplo1 as $b$

whereST_Intersects (b.geom, ST_Buffer(a.geom, 31))

Figura 11 - Parte do resultado da consulta para selecionar os pontos que possuem IRI médio acima de 3,5 e que possuem algum vizinho a até $21 \mathrm{~m}$ com valor também acima de 3,5 .

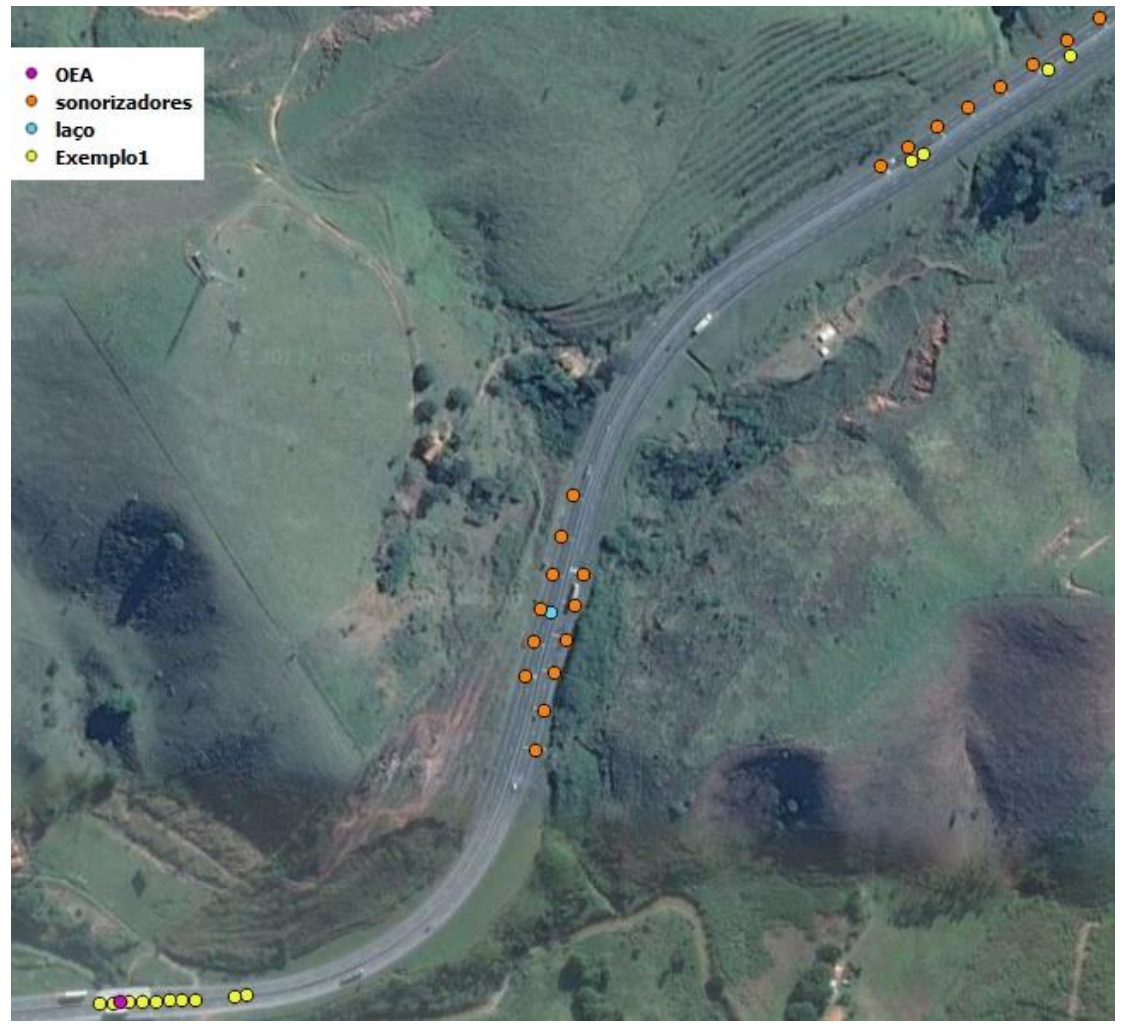

Figura 12 - Resultado da Figura $11 \mathrm{com}$ apenas os pontos que estão a até $31 \mathrm{~m}$ de uma OEA.

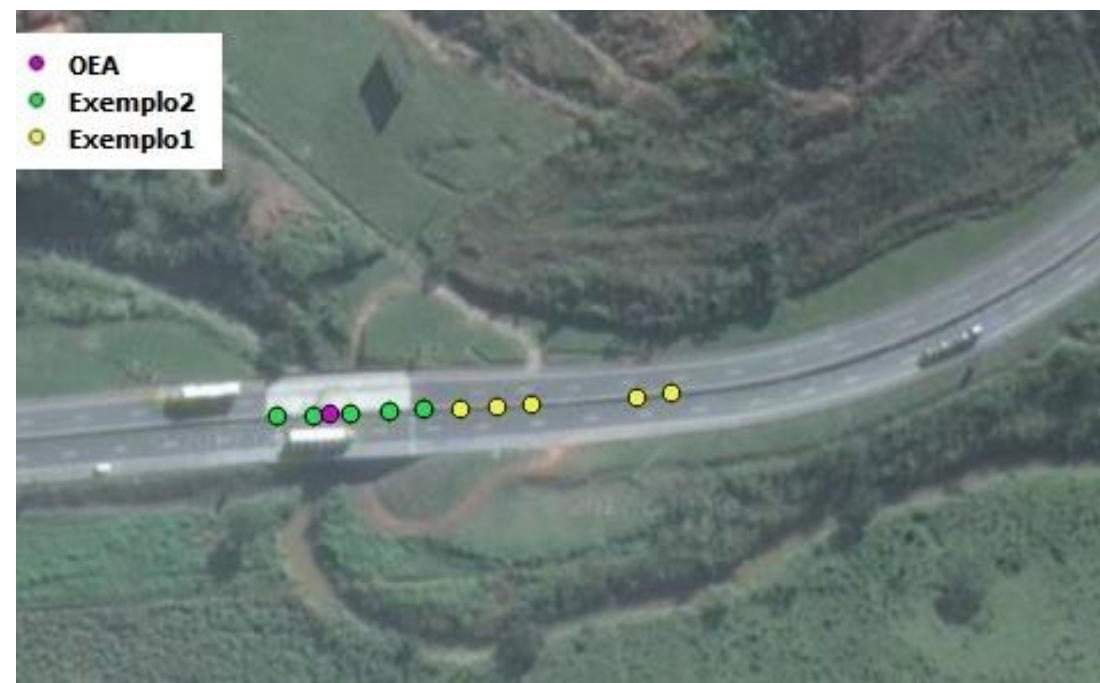


Exemplo 3: Selecionar apenas os pontos que estão a certa distância de um local de interesse. Na consulta a seguir são listados apenas os pontos da faixa 1 que estão a até $100 \mathrm{~m}$ de um laço.

select a.*

from tbf1_10 as a, tblaco as b

whereST_Intersects (a.geom, ST_Buffer(b.geom, 101))

\section{Discussão}

Ao utilizar um ambiente de SIG para fazer as análises da qualidade dos pavimentos de rodovias, tem-se a possibilidade de adicionar funcionalidades que não estão disponíveis fora de um ambiente espacial. Além disso, não é possível utilizar as informações de coordenadas geográficas coletadas pelos equipamentos de leitura da qualidade do pavimento.

Ao longo do Capítulo de Resultados foram apresentados alguns exemplos de aplicações de um ambiente de SIG nos dados de qualidade de pavimento e a seguir tem-se alguns pontos a serem destacados:

a) Visualização espacial dos dados - dados tabulares são apenas números, já quando plotados num mapa, assim como no exemplo da Figura 8, eles possuem forma e cor, podem ser melhor interpretados e associados a outros elementos, tal como, presença de curvas;

b) Comparação entre dados - ao comparar dois dados de IRI num ambiente tabular, tem-se apenas comparações numéricas, já num SIG é possível também fazer comparações visuais, tome como exemplo os casos da Figura 9 e Figura 10. No mapa a visualização é mais simples e fica mais fácil perceber similaridades nos dados;

c) Associação entre dados -num ambiente tabular as operações entre os dados são apenas numéricas, isto é, operações tipicamente espaciais não estão disponíveis, tais como, operações de vizinhança (detectar os vizinhos de cada ponto), interseção ou proximidade entre dados distintos (comparar leituras que estão em faixas ou mesmo pistas distintas, mas que estão espacialmente próximos);

d) Potencialidades das operações espaciais - as operações espaciais podem abrir diversas possibilidades de cruzamento de dados para auxiliar na gestão do pavimento das rodovias, como exemplo: aumento de trechos urbanos, volume de trafego,dados de acidentes, dados de elevação coletados pelo perfilômetro, dados históricos, etc.

\section{Conclusão}

Pela própria característica espacial dos dados de qualidade de pavimento a sua análise se mostrou apropriada num ambiente de SIG, já que os dados especializados num mapa propiciam melhor contextualização. Além 
disso, operações espaciais entre os dados de qualidade de pavimento e também incluindo dados externos evidenciam e ajudam a explicar características nos índices de qualidade de pavimento. Um ambiente de SIG poderia ainda oferecer mais benefícios se inserisse uma série temporal de leituras de qualidade do pavimento, além de datas e características de restauração. Desta forma, seria possível mapear degradação do pavimento levando em conta fatores externos e históricos.

\section{Agradecimentos}

Agradeço imensamente à Deus, por ter me concedido saúde, força e disposição durante minha gestação para finalizar o curso de tecnólogo em Geoprocessamento.

Ao meu orientador, prof. Dr. Arley Ferreira de Souza, pelos conselhos e ensinamentos, auxiliando-me na concretização dessa pesquisa.

Aos professores e funcionários da Faculdade Tecnológica de Jacareí (FATEC), pelos conhecimentos transmitidos.

Ao Centro de Pesquisas Rodoviárias (CPR)- CCR Nova Dutra pela concessão dos dados de IRI, em especial a Coord. a Eng. ${ }^{\text {a }}$ Valéria Cristina Faria pelo incentivo e apoio à pesquisa.

A toda minha família e amigos, principalmente à Cláudia Aparecida Macedo, minha parceira de estudo, e ao meu esposo Geraldo Pereira.

\section{Referências}

BENEVIDES, S. A. S.; SILVA, B. T. A.; OLIVEIRA, F. H. L. Análise Comparativa Dos Valores Do Índice Internacional de Irregularidade em Rodovias do Estado do Ceará, 15a Reunião de Pavimentação Urbana, Trabalho $\mathrm{n} \div 25, \quad$ Salvador/BA, 2008. Disponível em:<http://www.berater.com.br/wp-content/uploads/2014/02/Artigo-DefinitivoAn+\%C3\%ADlise-Comparativa-IRI-15aRPU.pdf>. Acesso em: 12/04/2017.

BENEVIDES, S. A. S.; MOTTA, L. M. G. Comparação dos valores de irregularidade longitudinal medidos com o perfilômetro a laser com variação do arranjo das posições dos sensores. TRANSPORTES, v. XVIII, n. 2, p. 72-80, junho $2010 . \quad$ Disponível em: <https://www.revistatransportes.org.br/anpet/article/view/426>. Acesso em: 09/08/2017.

Cibermétrica, $2017 \quad-\quad-15$ Disponível <http://www.cibermetrica.com.br/CiberLaser.html>. Acesso em: 30/09/2017.

CNT (Confederação Nacional de Transportes). Transporte rodoviário: Por que os pavimentos das rodovias do Brasil não duram. Brasília/DF, 2017. Disponível 
em:

<http://cms.cnt.org.br/Imagens\%20CNT/PDFs\%20CNT/Estudos\%20CNT/estud o_pavimentos_nao_duram.pdf>. Acesso em: 08/082017.

DNIT (Departamento Nacional de Infraestrutura de Transportes). Manual de Restauração de Pavimento Asfálticos, 2. Ed., Rio de Janeiro/RJ, 2006. Disponível em: <http://www1.dnit.gov.br/ipr_new/..\%5Carquivos_internet\%5Cipr\%5Cipr_new\% 5Cmanuais\%5CManual_de_Restauracao.pdf>. Acesso em: 08/08/2017.

DNIT (Departamento Nacional de Infraestrutura de Transportes). Manual de gerência de pavimentos, 189p. (IPR. Publ. 745), Rio de Janeiro, 2011. Disponível em: <http://ipr.dnit.gov.br/normas-emanuais/manuais/documentos/745_manual_de_gerencia_de_pavimentos.pdf $>$. Acesso em: 15/08/2017.

ELHADI, H. M. A. Gis a Tool for Pavement Management, Royal Institute of Technology (KTH), Estocolmo/Suécia,2009. Disponível em: <https://www.divaportal.org/smash/get/diva2:1064849/FULLTEXT01.pdf>. Acesso em: 16/02/2016.

FORSLOF, L. Roughness and Texture, 2014. Disponível em: <https://www.linkedin.com/pulse/20141130211746-97325448-roughness-andtexture >. Acesso em: 12/02/2016.

GRASS, D. M. Integration of GIS into pavement management systems for low volume county roads. University Auburn, Auburn/Alabama, 2007. Disponível em: <http://etd.auburn.edu/handle/10415/68>. Acesso em: 16/12/2016.

IBRAHEEM, A. T.; FALIH, D. A. A. Applying Geographic Information System (GIS) for Maintenance Strategy Selection, Scientific Research, Vol. 4 No. 1 (2012), Article ID: 17099, 11 pages. Disponível em: <http://file.scirp.org/html/78101538_17099.htm>. Acesso em: 16/12/2016.

RODRIGUES, C. M. A.; Modelos de previsão da degradação de pavimentos: parâmetro IRI. Dissertação (Mestrado), Faculdade de Ciências e Tecnologia da Universidade Nova de Lisboa, Lisboa, 2013.148p. Disponível em: http://hdl.handle.net/10362/11233. Acesso em: 01/10/2017.

SAYERS, M. W.; GILLESPIE, T. D.; QUEIROS, C. A. V. The International Road Roughness Experiment: Establishing Correlation and a Calibration Standard for Measurements, World Bank technical paper, ISSN 0253-7494; no. 45, Washington/DC, $1986 . \quad$ Disponível em: <http://documents.worldbank.org/curated/pt/326081468740204115/TheInternational-Road-Roughness-Experiment-IRRE-establishing-correlation-anda-calibration-standard-for-measurements>. Acesso em: 08/10/2017. 
SEVERO, L. E. P.; RUWER, P.; KLEIN, R. J.; BARELLA, R. M.; MASSARA, S.; GONÇALVES, F. P. Utilização de perfilômetro laser para a medida de irregularidade longitudinal e do afundamento em trilhas de roda. 9 Encontro Nacional de Conservação Rodoviária, Natal/RN, 2004. Disponível em: <http://pavesys.com.br/download/27\%20\%20Utiliza\%C3\%A7\%C3\%A30\%20de\%20Perfil\%C3\%B4metro\%20Laser\%20p ara\%20a\%20medida\%20de\%20Q1\%20e\%20ATR.pdf>. Acesso em: 01/10/2017.

VOGEL, C. R. acompanhamento da irregularidade longitudinal pós-restauração de pavimento em concreto asfáltico de rodovia estadual do noroeste do Estado do Rio Grande do Sul. Universidade Federal do Estado do Rio Grande do Sul, Santa Rosa/RS, 2015.2 Disponível em: $<$ http://bibliodigital.unijui.edu.br:8080/xmlui/handle/123456789/3406>. Acesso em: 01/10/2017.

ZHOU, G. Co-Location decision tree for enhancing decision-making of pavement maintenance and rehabilitation. Virginia Polytechnic Institute And State University, Blacksburg/ Virginia, 2011.Disponível em: <https://www.sciencedirect.com/science/article/pii/S0968090X11001513>. Acesso em: 16/12/2016. 